\title{
Collisional Isotropisation of a Strongly Magnetized Anisotropic Plasma within the Weak Interaction Approximation
}

\author{
H. Schamel and G. Hübner \\ Institute of Theoretical Physics IV, University of Bayreuth, W-8580 Bayreuth, Germany
}

Z. Naturforsch. 47a, 629-636 (1992); received December 31, 1991

The equipartition frequency $v$ of an anisotropic plasma is calculated numerically and analytically within the Landau approach for strong magnetic fields and several new integral expressions for $v$ are presented. In the ultra strong magnetic field case, when $r_{\mathrm{C}} \ll b$, where $r_{\mathrm{C}}$ is the Larmor radius and $b$ the classical distance of closest approach, $v$ is shown to be dependent on how the Coulomb force is cut off at short distances. None of the obtained expressions, however, coincides with experiment, in contrast to the strong interaction theory of O'Neil and Hjorth. For weaker, but still strong magnetic fields $\left(r_{\mathrm{C}}>b\right)$ the ambiguity disappears and the weak interaction result describes well the experiment. It is, hence, the transition region $r_{\mathrm{C}} \approx b$, where the transition from strong to weak interaction takes place.

Key words: Landau collision operator, Collision frequency, Magnetized plasma, Weak coupling theory, Strong coupling theory.

\section{Introduction}

The collisional relaxation of anisotropic temperatures $T_{\perp}$ and $T_{\|}$of a weakly correlated plasma that is immersed in a uniform magnetic field $\boldsymbol{B}=B \hat{b}$ is known to depend on the strength of the magnetic field [1-4]. Taking into account self-collisions only and exploiting the weak-interaction approximation (Landau approach), three regions of the magnetic field dependence of the equipartition collision frequency $v$ can be distinguished.

In region $\mathrm{I}$, when the thermal Debye length $\lambda_{\mathrm{D}}$ is smaller than the Larmor radius $r_{\mathrm{C}}$, the equipartition frequency $v$ is found to be independent of $B\left(\lambda_{\mathrm{D}}=\right.$ $\sqrt{\varepsilon_{0} T_{0} / n e^{2}}$, where $T_{0}=\left(T_{\|}+2 T_{\perp}\right) / 3$ is the isotropic temperature of the considered species; $r_{\mathrm{C}}=$ $\sqrt{T_{0} / m} \Omega^{-1}$, where $\Omega=e B / m$ is the Larmor frequency).

In the intermediate region II, given by $b<r_{\mathrm{C}}<\lambda_{\mathrm{D}}$, a logarithmic decrease of $v$ is found with increasing $B$ $\left(b \doteq e^{2} /\left(12 \pi \varepsilon_{0} T_{0}\right)\right.$ is the Landau length or classical distance of closest approach).

In the strong magnetic field region III, where $r_{\mathrm{C}}<b$ holds, an extremely small collision frequency is obtained.

Present day fusion plasmas like JET or the solar wind plasma $[5,6]$ are found to lie in region $\mathrm{I}$; the

Reprint requests to Dr. H. Schamel, Universität Bayreuth, Theoretische Physik IV, Postfach 1012 51, W-8580 Bayreuth, Germany. magnetic field has, therefore, little influence on the equipartition rate (in JET plasmas this is well satisfied for ions and marginally true for electrons $\left(r_{\mathrm{Ce}} \approx \lambda_{\mathrm{De}}\right)$, whereas in the solar wind both species well satisfy the criterion $r_{\mathrm{Cs}}>\lambda_{\mathrm{Ds}}$ for $s=e, i$ ).

Recent experimental progresses in confining a weakly correlated, pure electron plasma $[7,8]$ have now made accessible regions II and III and, hence, provide a test of analytic expressions derived for $v$ in this parameter regime. In these experiments the criterion for the validity of a classical treatment of the collision dynamics, namely, $\hbar \Omega<T_{0}<5 \mathrm{eV}[9,10]$, was satisfied.

Within the Landau theory a weakly correlated plasma is assumed, i.e., $\Lambda \doteq \lambda_{\mathrm{D}} / b=12 \pi n \lambda_{\mathrm{D}}^{3} \gg 1$, where $\Lambda$ is the quantity appearing in the Coulomb logarithm, $\ln \Lambda$, and it holds $\Lambda=12 \pi g^{-1}$, where $g \doteq\left(n \lambda_{\mathrm{D}}^{3}\right)^{-1} \ll 1$ is the plasma parameter. The decrease of $v$ with increasing $B$ in region II is approximately described by a replacement of $\Lambda_{\mathrm{De}}$ with $r_{\mathrm{Ce}}$ in the Coulomb logarithm. This can be interpreted as a shortening of the effective interaction range perpendicular to $\boldsymbol{B}$ from $\lambda_{\mathrm{D}}$ to $r_{\mathrm{C}}$. It also shows that distant encounters, i.e., small-angle collisions between particles with impact parameters $\varrho$ in the order of $\lambda_{\mathrm{D}}$ become less effective.

On the other hand, referring to the ultra strong magnetic field case, $r_{\mathrm{C}} \ll b$, O'Neil found that the most effective collisions in this region are close encounters, i.e., collisions with $\varrho \approx b$. He realized that the motion 
of two such colliding particles is controlled by an adiabatic invariant, $u_{\perp}^{2}$, where $\boldsymbol{u}_{\perp}$ is the relative velocity of the particles perpendicular to the homogeneous magnetic field, and developed a strong interaction theory by exploiting the Boltzmann collision integral $[10,11]$. This adiabatic invariant implies that the exchange of perpendicular energy or the scattering of the perpendicular velocity components, respectively, is governed by a dipole-like rather than a monopolelike interaction and, hence, a Boltzmann-type operator can be justified from the BBGKY hierarchy due to this more short range interaction. The essence of this adiabatic invariant is that it is conserved only asymptotically, when $r_{\mathrm{C}} / b \rightarrow 0$ and that exponentially small changes in perpendicular energy will take place when $B$ is large but finite. Within the strong interaction theory [10], the equipartition frequency is, therefore, expected to drop off exponentially.

However, in cases of weaker magnetic fields - more precisely in the regime of interest representing the transition from region III to region II, $r_{\mathrm{C}} \approx b-$ the adiabatic expansion breaks down and it becomes questionable whether the close encounters $\varrho \approx b$ contribute most effectively. So, one is forced to conclude that at present no convincing theory is available for this transition region. Here, neither the unperturbed helices upon which the Landau- or small interactionapproach is based nor the $\boldsymbol{E} \times \boldsymbol{B}$ guiding center motion, where $\boldsymbol{E}$ is the microelectric field of the point charge, which is the basis of the strong interaction theory [10], are believed to be meaningful tools in describing the collision dynamics.

In such an unsatisfactory situation it seems meaningful that one evaluates each approach as exactly as possible to be sure that the differences to a more sophisticated theory, which may arise in the future, will be due to an incomplete treatment of the collision processes of the former theories and will not be further masked by an imprecise or doubtful calculation of the collision frequency within each approach. Another possibility to test these approaches are Monte-Carlo simulations with many particles $[12,13]$.

The present paper represents a continuation and deepening of our earlier one [4]. Relying on the Landau approach, it focusses on the strong magnetic field regime, $r_{\mathrm{C}} \leqq b$, i.e., on a regime, where the formula in [3] for $v$ ceases to be valid. We present several forms of the general integral expression for the equipartition collision frequency $v$, determine the behavior of $v$ in the transition region in more detail and show by a formal expansion of $v$ for the ultra strong magnetic field case, $r_{\mathrm{C}} \ll b$ that in this case the Landau approach itself is no longer uniquely defined but depends on how the cut-off is introduced into the Coulomb force. In general no restriction on the strength of the temperature anisotropy is imposed. Furthermore, comparisons are made with recent experimental data [8].

The paper is organized as follows. In Sect. 2 the weak interaction formalism is presented in the framework of [3] and the matrix elements are exposed, which sensitively will depend on how the cut-off is introduced. Section 3 is devoted to an evaluation of the equipartition frequency, and the above mentioned comparisons of the two theories with experiment are made in Section 4. Furthermore, in the appendix a new form of the asymptotic expansion of Bessel functions is presented.

\section{Derivation of Basic Equations}

The kinetic equation, we are starting with, is of Landau-Rostoker-type $[14,15]$ and is found, e.g., in [3], which also serves as a guide line. It is valid in the weak interaction approximation, treats, hence, only distant encounters correctly (small angle scattering), ignores relativistic, quantum, polarisation, nonuniform and non-Markovian effects and reads for the $s$-th species

$$
\begin{gathered}
{\left[\partial_{t}+\frac{q_{s}}{m_{s}} \boldsymbol{v}_{1} \times \boldsymbol{B} \cdot \partial \boldsymbol{v}_{1}\right] f^{s}\left(\boldsymbol{v}_{1}, t\right)=-\partial_{\boldsymbol{v}_{1}} \cdot \boldsymbol{J}^{s}\left(\boldsymbol{v}_{1}, t\right),} \\
\boldsymbol{J}^{s}=-\frac{1}{m_{s}} \sum_{s^{\prime}} \int \mathrm{d} \boldsymbol{r}_{12} \mathrm{~d} \boldsymbol{v}_{2} \int_{-\infty}^{0} \mathrm{~d} \tau \frac{\partial}{\partial \boldsymbol{r}_{12}} \phi\left(\boldsymbol{r}_{12}\right) \frac{\partial \phi\left(\boldsymbol{r}_{12}(\tau)\right)}{\partial \boldsymbol{r}_{12}(\tau)} \\
\cdot\left[\frac{1}{m_{s}} \frac{\partial}{\partial \boldsymbol{v}_{1}(\tau)}-\frac{1}{m_{s^{\prime}}} \frac{\partial}{\partial \boldsymbol{v}_{2}(\tau)}\right] f^{s}\left(\boldsymbol{v}_{1}(\tau), t\right) f^{s^{\prime}}\left(\boldsymbol{v}_{2}(\tau), t\right),
\end{gathered}
$$

where the notation is standard [15], $\phi$ represents the electrostatic Coulomb potential

$$
\phi\left(\boldsymbol{r}_{12}\right)=\frac{q_{s} q_{s^{\prime}}}{4 \pi \varepsilon_{0}\left|\boldsymbol{r}_{12}\right|},
$$

$\boldsymbol{r}_{12}$ is the relative position vector of two colliding particles

$$
\boldsymbol{r}_{12}=\boldsymbol{r}_{1}-\boldsymbol{r}_{2}
$$

and $\boldsymbol{r}_{i}(\tau), \boldsymbol{v}_{i}(\tau)$ stands for the $i$-th particle unperturbed orbit at time $\tau$. 
Two further simplifiations are adopted. We consider only like-particle collisions, setting $s=s^{\prime}=e, q_{e}=-e$, $m_{e} \equiv m$, and assume a gyrotropic electron distribution $f^{\mathrm{e}}(\boldsymbol{v}(\tau), t) \equiv f\left(v_{\perp}^{2}(\tau), v_{\|}^{2}(\tau), t\right)$ which is, hence, independent of the gyroangle of an electron gyrating around the magnetic field line.

The free particle trajectories are determined by the equations of motion (the characteristics of the Vlasov operator)

$$
\begin{aligned}
\dot{\boldsymbol{r}}_{i}(\tau) & =\boldsymbol{v}_{i}(\tau), \\
\dot{\boldsymbol{v}}_{i}(\tau) & =\frac{q_{\mathrm{e}}}{m_{\mathrm{e}}} \boldsymbol{v}_{i}(\tau) \times \boldsymbol{B} \equiv \Omega\left[\hat{b} \times \boldsymbol{v}_{i}(\tau)\right],
\end{aligned}
$$

where $\Omega \equiv e B / m$ is the electron gyrofrequency. Equation (4) yields upon integration

$\boldsymbol{r}_{i}(\tau)=\boldsymbol{r}_{i}+\boldsymbol{v}_{i \perp} \frac{\sin \Omega \tau}{\Omega}-\hat{b} \times \boldsymbol{v}_{i \perp} \frac{\cos \Omega \tau-1}{\Omega}+\hat{b} v_{i \|} \tau$,

$\boldsymbol{v}_{i}(\tau)=\boldsymbol{v}_{i \perp} \cos \Omega \tau+\hat{b} \times \boldsymbol{v}_{i \perp} \sin \Omega \tau+\hat{b} v_{i \|}$.

Using $\frac{\partial}{\partial \boldsymbol{v}_{\perp}(\tau)}=\frac{\boldsymbol{v}_{\perp}(\tau) \boldsymbol{v}_{\perp}}{v_{\perp}^{2}} \cdot \frac{\partial}{\partial \boldsymbol{v}_{\perp}}$, valid for gyrotropic distributions, $\boldsymbol{J}^{\mathrm{e}} \equiv \boldsymbol{J}$ becomes

$$
\begin{aligned}
\boldsymbol{J}= & -\frac{1}{m^{2}} \int \mathrm{d} \boldsymbol{r}_{12} \mathrm{~d} \boldsymbol{v}_{2} \frac{\partial \phi\left(\boldsymbol{r}_{12}\right)}{\partial \boldsymbol{r}_{12}} \int_{-\infty}^{0} \mathrm{~d} \tau \frac{\partial \phi\left(\boldsymbol{r}_{12}(\tau)\right)}{\partial \boldsymbol{r}_{12}(\tau)} \\
& \cdot\left[\left(\frac{\boldsymbol{v}_{1 \perp}(\tau) \boldsymbol{v}_{1 \perp}}{v_{1 \perp}^{2}}+\frac{\boldsymbol{v}_{1 \|}(\tau) \boldsymbol{v}_{1 \|}}{v_{1 \|}^{2}}\right) \cdot \frac{\partial}{\partial \boldsymbol{v}_{1}}-(1 \rightarrow 2)\right] \\
& \cdot f\left(v_{1 \perp}^{2}, v_{1 \|}^{2}, t\right) f\left(v_{2 \perp}^{2}, v_{2 \|}^{2}, t\right) .
\end{aligned}
$$

Next, we introduce the Fourier-Transformation

$$
\phi(\boldsymbol{r})=\int \mathrm{d} \boldsymbol{k} \hat{\phi}(\boldsymbol{k}) e^{i \boldsymbol{k} \cdot \boldsymbol{r}},
$$

so that $\phi(\boldsymbol{r})=e^{2} / 4 \pi \varepsilon_{0} r$ transforms to $\hat{\phi}(\boldsymbol{k})=e^{2} /$ $\left(8 \pi^{3} \varepsilon_{0} k^{2}\right)$.

$\boldsymbol{J}$ can then be written as

$\boldsymbol{J}=\int \mathrm{d} \boldsymbol{v}_{2} Q\left(\boldsymbol{v}_{1}, \boldsymbol{v}_{2}\right) \cdot\left(\frac{\partial}{\partial \boldsymbol{v}_{1}}-\frac{\partial}{\partial \boldsymbol{v}_{2}}\right) f\left(\boldsymbol{v}_{1}, t\right) f\left(\boldsymbol{v}_{2}, t\right)$,

where the tensor $Q$ is given by

$Q=-\frac{(2 \pi)^{3}}{m^{2}} \int \mathrm{d} \boldsymbol{k}|\hat{\phi}(\boldsymbol{k})|^{2} \int_{-\infty}^{0} \mathrm{~d} \tau \boldsymbol{k} \boldsymbol{k}(\tau) e^{i \boldsymbol{k} \cdot \Delta X_{12}(\tau)+\varepsilon \tau}$

with the definitions

$$
\begin{aligned}
\Delta X_{12}(\tau) & \doteq \boldsymbol{v}_{12} \perp \frac{\sin \Omega \tau}{\Omega}-\hat{b} \times \boldsymbol{v}_{12 \perp} \frac{\cos \Omega \tau-1}{\Omega}+\hat{b} v_{12 \|} \tau, \\
\boldsymbol{k}(\tau) & \doteq \boldsymbol{k}_{\perp} \cos \Omega \tau+\boldsymbol{k}_{\perp} \times \hat{b} \sin \Omega \tau+\boldsymbol{k}_{\|} .
\end{aligned}
$$

In the limit $\Omega \rightarrow 0$ the usual Landau operator is easily obtained:

$$
\begin{aligned}
& \boldsymbol{k}(\tau) \rightarrow \boldsymbol{k} \\
& \Delta X_{12}(\tau) \rightarrow \boldsymbol{v}_{12} \tau \\
& \int_{-\infty}^{0} \mathrm{~d} \tau e^{i \boldsymbol{k} \cdot \boldsymbol{v}_{12} \tau+\varepsilon \tau}=\frac{1}{i\left(\boldsymbol{k} \cdot \boldsymbol{v}_{12}-i \varepsilon\right)} \\
& =\frac{1}{i}\left[P \frac{1}{\boldsymbol{k} \cdot \boldsymbol{v}_{12}}+i \pi \delta\left(\boldsymbol{k} \cdot \boldsymbol{v}_{12}\right)\right], \\
& Q=\frac{-(2 \pi)^{3} \pi}{m^{2}} \int \mathrm{d} \boldsymbol{k}|\hat{\phi}(k)|^{2} \boldsymbol{k} \boldsymbol{k} \delta\left(\boldsymbol{k} \cdot \boldsymbol{v}_{12}\right) \\
& =\frac{-8 \pi^{5}}{m_{\mathrm{e}}^{2}} \int \mathrm{d} k k^{3}|\hat{\phi}(k)|^{2} \frac{1}{u^{3}}\left(u^{2} \mathscr{I}-\boldsymbol{u} \boldsymbol{u}\right),
\end{aligned}
$$

where $\boldsymbol{u} \equiv \boldsymbol{v}_{12}=\boldsymbol{v}_{1}-\boldsymbol{v}_{2}$ and $\mathscr{I}$ is the unit tensor.

Introducing $\hat{\phi}(\boldsymbol{k})$ in the second step of the last equation, the $\boldsymbol{k}$-integral in $Q$ reduces (up to a constant) to $\int_{k_{\mathrm{D}}}^{k_{0}} \mathrm{~d} k k^{-1}=\ln k_{0} / k_{\mathrm{D}} \equiv \ln \lambda_{\mathrm{D}} / b=\ln \Lambda$, where the usual cut-offs are introduced to avoid singularities. $\left(k_{\mathrm{D}} \doteq \lambda_{\mathrm{D}}^{-1}, k_{0} \doteq b^{-1}\right)$. This shows how the Coulomb logarithm comes into play.

For finite $\Omega$, it can be shown that only the symmetric part of $Q$ contributes and that its tensor elements can be expressed by $Q_{11} \equiv \hat{e}_{1} \cdot Q \cdot \hat{e}_{1}$, where $\hat{e}_{1}$ is a unit vector perpendicular to $\hat{b}=\boldsymbol{B} / \boldsymbol{B}$. The wave vector is then decomposed either cylindrically into

$$
\boldsymbol{k}=\hat{e}_{1} k_{\perp} \cos \alpha+\hat{e}_{2} k_{\perp} \sin \alpha+\hat{b} k_{\|}
$$

or spherically into

$\boldsymbol{k}=\hat{e}_{1} k \sin \theta \cos \alpha+\hat{e}_{2} k \sin \theta \sin \alpha+\hat{b} k \cos \theta$,

where $\alpha$ and $\theta$ are the azimuthal and polar angle, respectively $\left(\hat{e}_{2}=\hat{b} \times \hat{e}_{1}\right)$.

The tensor element $Q_{11}$ then reads

$$
\begin{aligned}
& Q_{11}=-\frac{(2 \pi)^{3}}{m^{2}} \int \mathrm{d} \boldsymbol{k}|\hat{\phi}(k)|^{2} \int_{-\infty}^{0} \mathrm{~d} \tau \\
& \cdot \exp \left(\varepsilon \tau+i\left\{\frac{k_{\perp} u_{\perp}}{\Omega}[\sin (\Omega \tau-\alpha)+\sin \alpha]\right.\right. \\
&\left.\left.\quad+k_{\|} u_{\|} \tau\right\}\right) k_{\perp}^{2} \cos \alpha \cos (\alpha-\Omega \tau) .
\end{aligned}
$$

It depends on how the $\boldsymbol{k}$-integral is treated, i.e., whether a cut-off is introduced in the $k_{\perp}$-integration only (case A) or in the spherical $k$-integration (case B). In the latter case also a $k_{\|}$-cut-off is implied. In both 
cases use is made of the Bessel function expansion

$$
\exp [i z \sin \Phi]=\sum_{l=-\infty}^{+\infty} J_{l}(z) e^{i l \Phi} .
$$

\section{Case A: Cylindrical Cut-Off}

Treating the $\boldsymbol{k}$-integral in a cylindrical manner, $\int \mathrm{d} \boldsymbol{k}=\int \mathrm{d} k_{\perp} k_{\perp} \int_{-\infty}^{+\infty} \mathrm{d} k_{\|} \int_{0}^{2 \pi} \mathrm{d} \alpha$, and performing the $\tau$ and $\alpha$-integrations, one obtains

$$
\begin{aligned}
Q_{11}= & -\frac{(2 \pi)^{4} \pi}{m^{2}} \sum_{l=-\infty}^{+\infty} \int \mathrm{d} k_{\perp} k_{\perp}^{3} \int_{-\infty}^{+\infty} \mathrm{d} k_{\|}|\hat{\phi}(k)|^{2} \\
& \cdot\left[\frac{l}{\xi} J_{l}(\xi)\right]^{2} \delta(l \Omega+\zeta),
\end{aligned}
$$

where $\xi \doteq k_{\perp} u_{\perp} / \Omega, \zeta \doteq k_{\|} u_{\|}$.

Introducing $k^{2}=k_{\perp}^{2}+k_{\|}^{2}$ in $\hat{\phi}(k)$ and performing the $k_{\|}$-integral, $Q_{11}$ then becomes

$$
\begin{aligned}
Q_{11}= & -\frac{e^{4}}{4 \pi \varepsilon_{0}^{2} m^{2}} \frac{1}{\left|u_{\|}\right|} \sum_{l=-\infty}^{+\infty} \int \mathrm{d} k_{\perp} \frac{k_{\perp}^{3}}{\left[k_{\perp}^{2}+\left(\frac{l \Omega}{u_{\|}}\right)^{2}\right]^{2}} \\
& \cdot\left[\frac{l \Omega}{k_{\perp} u_{\perp}} J_{l}\left(\frac{k_{\perp} u_{\perp}}{\Omega}\right)\right]^{2},
\end{aligned}
$$

where the boundaries in the $k_{\perp}$-integral still have to be specified.

\section{Case B: Spherical Cut-Off}

If we treat, on the other hand, the $k$-integral spherically, $\int \mathrm{d} \boldsymbol{k}=\int \mathrm{d} k k^{2} \int_{0}^{\pi} \sin \theta \mathrm{d} \theta \int_{0}^{2 \pi} \mathrm{d} \alpha$ and perform the $\tau$ and $\alpha$-integrations, we get

$$
\begin{aligned}
Q_{11}= & -\frac{e^{4}}{4 \pi \varepsilon_{0}^{2} m^{2}} \sum_{l=-\infty}^{+\infty} \int \mathrm{d} k \int_{0}^{\pi} \mathrm{d} \theta \sin ^{3} \theta \\
& \cdot\left[\frac{l \Omega}{k u_{\perp} \sin \theta} J_{l}\left(\frac{k u_{\perp} \sin \theta}{\Omega}\right)\right]^{2} \delta\left(l \Omega+k u_{\|} \cos \theta\right),
\end{aligned}
$$

where it is the $k$-integration which has yet to be cutoff.

The temperature isotropisation can be treated for both cases in the following way.

With the definitions of parallel and perpendicular energies,

$$
\begin{aligned}
& W_{\|}=\int \mathrm{d} \boldsymbol{v}_{1} \frac{m}{2} v_{1 \|}^{2} f \equiv \frac{n T_{\|}}{2}, \\
& W_{\perp}=\int \mathrm{d} \boldsymbol{v}_{1} \frac{m}{2} v_{1 \perp}^{2} f \equiv n T_{\perp},
\end{aligned}
$$

and with bi-Maxwellians for $f$ (the validity of which is analyzed in [16] $)^{2}$,

$f_{b M}(v)=n\left(\frac{m}{2 \pi}\right)^{3 / 2} \frac{1}{\sqrt{T_{\|}}} \frac{1}{T_{\perp}} \exp \left[-\frac{m}{2}\left(\frac{v_{\perp}^{2}}{T_{\perp}}+\frac{v_{\|}^{2}}{T_{\|}}\right)\right]$,

the time-derivative of $W_{\|}$can be written as

$\dot{W}_{\|}=\frac{n \dot{T}_{\|}}{2}=\int \mathrm{d} \boldsymbol{v}_{1} \frac{m}{2} v_{1 \|}^{2} \partial_{t} f=n m \int \mathrm{d} \boldsymbol{v}_{1} v_{1 \|} J_{\|}$,

where

$$
\begin{aligned}
J_{\|}=-m \int \mathrm{d} \boldsymbol{v}_{2}[ & Q_{31}\left(\frac{v_{11}-v_{21}}{T_{\perp}}\right)+Q_{32}\left(\frac{v_{12}-v_{22}}{T_{\perp}}\right) \\
& \left.+Q_{33}\left(\frac{v_{13}-v_{23}}{T_{\|}}\right)\right] f_{b M}\left(\boldsymbol{v}_{1}\right) f_{b M}\left(\boldsymbol{v}_{2}\right) .
\end{aligned}
$$

The two $v$-integrations are transformed by a center-ofmass transformation

$$
\begin{aligned}
\boldsymbol{u} & \doteq \boldsymbol{v}_{1}-\boldsymbol{v}_{2}, \\
\boldsymbol{t} & \doteq \frac{1}{2}\left(\boldsymbol{v}_{1}+\boldsymbol{v}_{2}\right)
\end{aligned}
$$

to a $\boldsymbol{u}$ - and $\boldsymbol{t}$-integration. Chosing a frame in which $\boldsymbol{u}$ has no $y$-component, the second term in (20) drops out, and making use of

$Q_{31}=-\frac{u_{\perp}}{u_{\|}} Q_{11} \quad$ and $\quad Q_{33}=\left(\frac{u_{\perp}}{u_{\|}}\right)^{2} Q_{11}$

we obtain after the $\boldsymbol{t}$-integration

$$
\begin{aligned}
\dot{W}_{\|}= & -\frac{m^{7 / 2} n^{2}}{8 \sqrt{\pi}} \frac{1}{\sqrt{T_{\|}} T_{\perp}^{2}} \\
& \cdot\left(\frac{T_{\perp}}{T_{\|}}-1\right) \int_{-\infty}^{+\infty} \mathrm{d} u_{\|} \int_{0}^{\infty} \mathrm{d} u_{\perp} u_{\perp}^{3} Q_{11} \\
& \cdot \exp \left[-\frac{m}{4}\left(\frac{u_{\perp}^{2}}{T_{\perp}}+\frac{u_{\|}^{2}}{T_{\|}}\right)\right] .
\end{aligned}
$$

Through the conserved energy law

$$
\frac{T_{\|}}{2}+T_{\perp} \equiv \frac{3}{2} T_{0}
$$

where $T_{0}$ is the final isotropic temperature, $\dot{W}_{\|}$can be replaced by $\dot{\varepsilon}$, where

$$
\varepsilon(t) \doteq \frac{T_{\perp}(t)}{T_{\|}(t)}
$$

2 This choice of the distribution function is suggested by the experiments $[7,8]$ and allows in a most simple way the study of temperature isotropisation. 
H. Schamel and G. Hübner - Collisional Isotropisation of a Strongly Magnetized Anisotropic Plasma

is the anisotropy parameter. It holds

$T_{\perp}=\frac{3 \varepsilon T_{0}}{1+2 \varepsilon}, \quad T_{\|}=\frac{3 T_{0}}{1+2 \varepsilon}$,

and

$$
\dot{\varepsilon}(t)=-\frac{1+2 \varepsilon}{n T_{\|}} \dot{W}_{\|} .
$$

\section{The Equipartition Frequency}

\subsection{Case A: Cylindrical Cut-Off}

In the cylindrical case A, inserting $Q_{11}$ from (23) and using

$$
\begin{aligned}
& \int_{-\infty}^{+\infty} \mathrm{d} u_{\|} \frac{\left|u_{\|}\right|^{3} e^{-\beta u_{\|}^{2}}}{\left(a+u_{\|}^{2}\right)^{2}}=U(2,1 ; \beta a), \\
& \int_{0}^{\infty} \mathrm{d} u_{\perp} u_{\perp} J_{l}^{2}\left(b u_{\perp}\right) e^{-\alpha u_{\perp}^{2}}=\frac{1}{2 \alpha} I_{l}\left(\frac{b^{2}}{2 \alpha}\right) e^{-b^{2} / 2 \alpha},
\end{aligned}
$$

where $U(2,1 ; \beta a)$ is Kummer's function (being a special case of confluent hypergeometric functions) and $I_{l}(x)$ is the modified Bessel function, we obtain

$$
\begin{aligned}
\dot{\varepsilon}(t)= & \frac{\sqrt{m \pi} e^{4} n}{\left(4 \pi \varepsilon_{0}\right)^{2}} \frac{(1+2 \varepsilon)(1-\varepsilon)}{T_{\|}^{3 / 2} T_{\perp}} \\
& \cdot \sum_{l=-\infty}^{+\infty} l^{2} \int \mathrm{d} k_{\perp} \frac{(l \Omega)^{2}}{k_{\perp}^{3}} U\left(2,1 ; \frac{l^{2} \varepsilon}{2 x}\right) I_{l}(x) e^{-x},
\end{aligned}
$$

where $x \doteq 2 T_{\perp} k_{\perp}^{2} /\left(m \Omega^{2}\right)$. Replacing the $k_{\perp}$-integration by the $x$-integration, it follows

$$
\begin{aligned}
\dot{\varepsilon}(t)= & \frac{\sqrt{\pi} e^{4} n}{\sqrt{m}\left(4 \pi \varepsilon_{0}\right)^{2}} \frac{(1+2 \varepsilon)^{5 / 2}(1-\varepsilon)}{\left(3 T_{0}\right)^{3 / 2}} \\
& \cdot \sum_{l=-\infty}^{+\infty} l^{2} \int \mathrm{d} x \frac{1}{x^{2}} U\left(2,1 ; \frac{l^{2} \varepsilon}{2 x}\right) I_{l}(x) e^{-x},
\end{aligned}
$$

Because the $l=0$ term vanishes and the remaining series is symmetric in $l \rightarrow-l$ we get

$$
\begin{aligned}
\dot{\varepsilon}(t)= & \frac{2 \sqrt{\pi} e^{4} n}{\sqrt{m}\left(4 \pi \varepsilon_{0}\right)^{2}} \frac{(1+2 \varepsilon)^{5 / 2}(1-\varepsilon)}{\left(3 T_{0}\right)^{3 / 2}} \\
& \cdot \sum_{l=1}^{\infty} l^{2} \int \mathrm{d} x \frac{1}{x^{2}} U\left(2,1 ; \frac{l^{2} \varepsilon}{2 x}\right) I_{l}(x) e^{-x},
\end{aligned}
$$

Finally, introducing the normalized time $\tau=v_{E} t$, where

$$
v_{E}=\frac{8 \sqrt{\pi} e^{4} n \ln \Lambda}{\sqrt{m\left(3 T_{0}\right)^{3}}\left(4 \pi \varepsilon_{0}\right)^{2}} \equiv \frac{2}{\sqrt{3}} v_{\text {Spitzer }},
$$

we obtain

$$
\dot{\varepsilon}(\tau)=v(B, \varepsilon)(1-\varepsilon)
$$

with

$$
\begin{aligned}
v(B, \varepsilon) \equiv & \frac{1}{4 \ln \Lambda}(1+2 \varepsilon)^{5 / 2} \\
& \cdot \sum_{l=1}^{\infty} l^{2} \int \mathrm{d} x \frac{1}{x^{2}} U\left(2,1 ; \frac{l^{2} \varepsilon}{2 x}\right) I_{l}(x) e^{-x} .
\end{aligned}
$$

This is the sought equipartition frequency, but we still have to introduce the cut-offs in $k_{\perp}$ and to exploite the formula.

The cut-off in $k_{\perp}, k_{\mathrm{D}} \doteq \lambda_{\mathrm{D}}^{-1}\left(T_{0}\right) \leqq k_{\perp} \leqq k_{0} \doteq b^{-1}$ becomes in the $x$-variable $x_{\mathrm{D}} \leqq x \leqq x_{0}$ with

$$
\begin{aligned}
& x_{\mathrm{D}} \doteq \frac{6 \varepsilon}{1+2 \varepsilon}\left(\frac{k_{\mathrm{D}} v_{\mathrm{th} 0}}{\Omega}\right)^{2} \text { and } \\
& x_{0} \doteq \frac{6 \varepsilon}{1+2 \varepsilon}\left(\frac{k_{\mathrm{D}} v_{\mathrm{th} 0}}{\Omega}\right)^{2}, \quad v_{\mathrm{th} 0}^{2} \doteq \frac{T_{0}}{m} .
\end{aligned}
$$

Other forms of $v(B, \varepsilon)$, avoiding the Bessel function expansion, can be obtained. One more version is

$$
\begin{aligned}
v(B, \varepsilon)= & \frac{\sqrt{2 \pi}(1+2 \varepsilon)^{5 / 2}}{4 \ln \Lambda} \frac{\mathrm{d}}{\mathrm{d} \varepsilon} \varepsilon \frac{\mathrm{d}}{\mathrm{d} \varepsilon} \\
& \cdot\left[\frac{1}{\sqrt{\varepsilon}} \int_{x_{\mathrm{D}}}^{x_{0}} \frac{\mathrm{d} x}{\sqrt{x}} \int_{0}^{\infty} \mathrm{d} y I\left(\frac{x y^{2}}{2 \varepsilon}\right) e^{-x(1-\cos y)}\right],
\end{aligned}
$$

where $I(x)=e^{x} \operatorname{erfc} \sqrt{x}$ and where it is understood that the operator $\mathrm{d} / \mathrm{d} \varepsilon$ does not work on the $\varepsilon$-dependent $x_{\mathrm{D}}(\varepsilon)$ and $x_{0}(\varepsilon)$.

Another form is

$v(B, \varepsilon)=\frac{2 \sqrt{\pi}}{4 \ln \Lambda}(1+2 \varepsilon)^{5 / 2} \varepsilon^{-1} \int_{x_{\mathrm{D}}}^{x_{0}} \mathrm{~d} x \frac{F(x, \varepsilon)}{x}$

with

$$
\begin{aligned}
F(x, \varepsilon)= & \int_{0}^{\infty} \mathrm{d} t \exp \left[-2 x \sin ^{2}\left(t \sqrt{\frac{\varepsilon}{2 x}}\right)\right] \\
& \cdot \int_{0}^{\infty} \mathrm{d} y y^{2}\left(y^{2}-1\right) e^{-y^{2}-2 y t},
\end{aligned}
$$

which in a relatively elegant way can be obtained by exploiting Hassan's equations [17] (see also [18]). It is used in [4] to get numerically $v(B, \varepsilon)$ for arbitrary magnetic field strength. The main result, as mentioned in the introduction, is that there are three regions of magnetic field dependence: 
(I) $v(B, \varepsilon) \approx v(0, \varepsilon)$ which is the magnetic field free collision frequency, valid for $\lambda_{\mathrm{D}}<r_{\mathrm{C}}$, where $r_{\mathrm{C}}$ is the redefined electron gyroradius $\left(r_{\mathrm{C}} \equiv \sqrt{T_{\perp} / m} \Omega^{-1}\right)$. (II) $v(B, \varepsilon) \approx \frac{\ln \left(r_{\mathrm{C}} / b\right)}{\ln \Lambda} v(0, \varepsilon)$, in the intermediate range $b<r_{\mathrm{C}}<\lambda_{\mathrm{D}}$ which essentially means that in the Coulomb logarithm the Debye length has to be replaced by the gyroradius $[2,3]$, and

(III) an asymptotic expression valid for $r_{\mathrm{C}} \ll b$.

The magnetic field-free collision frequency in region I is easily obtained from (35). For $\Omega \rightarrow 0, x_{0}$ and $x_{\mathrm{D}}$ tend to infinity and only large values of $l$ contribute. Using $I_{l}(x) e^{-x} \sim \frac{1}{\sqrt{2 \pi x}} e^{-l^{2} / 2 x}$ (see Appendix) and changing the sum over $l$ to an integral over $l$, one finds

$$
\begin{aligned}
v(0, \varepsilon)= & \frac{1}{4 \ln \Lambda}(1+2 \varepsilon)^{5 / 2} \\
& \cdot \frac{2}{\sqrt{\pi}} \ln \Lambda \int_{0}^{\infty} \mathrm{d} t \sqrt{t} U(2,1 ; t) e^{-t},
\end{aligned}
$$

where the integral can be replaced by $\frac{\sqrt{\pi}}{2} \frac{1}{(1-\varepsilon)^{2}}$ $[(2+\varepsilon) g(\varepsilon)-3]$ and $g(\varepsilon)$ is given by (31) of [16].

Hence, we get

$$
v(0, \varepsilon)=\frac{(1+2 \varepsilon)^{5 / 2}}{4(1-\varepsilon)^{2}}[(2+\varepsilon) g(\varepsilon)-3],
$$

which is identical to (30) of [16].

The formal asymptotic expression for an ultra strong magnetic field is obtained even more simply. For $\Omega \rightarrow \infty, x_{0}$ and $x_{\mathrm{D}}$ tend to zero and $I_{l}(x)$ can be replaced by $I_{l}(x) \approx(x / 2)^{l} / l$ !; that means, only the $l=1$ term contributes to lowest order.

Since $U(2,1 ; \varepsilon / 2 x) \stackrel{x \rightarrow 0}{\sim} 4 x^{2} / \varepsilon^{2}$, we easily find by integration the desired equipartition frequency valid in the region III for case A:

$$
v_{\mathrm{A}}(B, \varepsilon)=\frac{(1+2 \varepsilon)^{5 / 2}}{\ln \Lambda \varepsilon^{2}}\left(\frac{r_{\mathrm{C}}}{b}\right)^{4} .
$$

In (41) a term $\Lambda^{-4}$ has been dropped compared to unity (it is introduced by the lower cut-off). This expression was already obtained in [4] ${ }^{3}$ and predicts an algebraic decrease of $v$ with $\Omega^{-4}$ in the limit $\Omega \rightarrow \infty$.

3 In this paper there are several misprints. In (4) the $s$-integral has to be taken from 0 to $\infty, \Lambda$ defined on page 3 is given by $\Lambda=12 \pi g^{-1}$ and in (11) $\sqrt{1+\varepsilon_{\alpha}}$ has to be replaced by $\sqrt{1+2 \varepsilon_{\alpha}}$.

\subsection{Case B: Spherical Cut-Off}

Now we turn to a spherical cut-off and show that it yields a rather different $\Omega$-dependence for $B \rightarrow \infty$.

Inserting $Q_{11}$ for case $\mathrm{B},(16 \mathrm{~b})$, into (23) and performing the $u_{\|}$- and $u_{\perp}$-integrations we get for $\dot{\varepsilon}(t)$

$$
\begin{aligned}
\dot{\varepsilon}(t)= & \frac{\sqrt{m \pi} n e^{4}}{\left(4 \pi \varepsilon_{0}\right)^{2}} \frac{1+2 \varepsilon}{T_{\|}^{3 / 2} T_{\perp}}(1-\varepsilon) \\
& \cdot \sum_{l=-\infty}^{+\infty}(l \Omega)^{2} \int \mathrm{d} k \frac{1}{k^{3}} \int_{0}^{\pi} \frac{\mathrm{d} \theta \sin \theta}{|\cos \theta|} \\
& \cdot \exp \left\{\frac{l^{2} \varepsilon}{2[x(\theta)-x(\pi / 2)]}\right\} I_{l}(x(\theta)) e^{-x(\theta)},
\end{aligned}
$$

where $x(\theta) \doteq 2 T_{\perp} k^{2} \sin ^{2} \theta /\left(m \Omega^{2}\right)$.

In the ultrastrong magnetic field limit, $\Omega \rightarrow \infty$, it holds $x(\theta) \rightarrow 0$ and $I_{l}(x(\theta)) \rightarrow(x(\theta) / 2)^{l} / l !, e^{-x(\theta)}$ $\approx 1-x(\theta)$. To lowest order only the terms linear in $x(\theta)$ contribute. The angle integral can be evaluated asymptotically,

$$
\int_{0}^{\pi} \mathrm{d} \theta \frac{\sin ^{3} \theta}{|\cos \theta|} \exp \left(-\frac{\alpha}{\cos ^{2} \theta}\right) \approx \frac{e^{-\alpha}}{\alpha^{2}},
$$

where $\alpha=m \Omega^{2} /\left(4 T_{\|} k^{2}\right) \equiv a / k^{2} \gg 1$. The remaining $k$-integral is made convergent by introducing an upper cut-off $k_{0}=1 / b$, and again a lower cut-off is not needed. Its asymptotic expression becomes

$$
\int_{0}^{k_{0}} \mathrm{~d} k k^{3} e^{-a / k^{2}} \approx \frac{1}{2 a b^{6}} e^{-a b^{2}}
$$

which shows, using $a b^{2}=\varepsilon\left(b / r_{\mathrm{C}}\right)^{2} / 4$, that for large values of $B$ the evolution of $\varepsilon=T_{\perp} / T_{\|}$is governed by

$$
\begin{aligned}
\dot{\varepsilon}(t)= & \frac{4 \sqrt{\pi} n e^{4}}{\sqrt{m}\left(3 T_{0}\right)^{3 / 2}\left(\pi \varepsilon_{0}\right)^{2}} \frac{(1+2 \varepsilon)^{5 / 2}}{\varepsilon^{3}}(1-\varepsilon)\left(\frac{r_{\mathrm{C}}}{b}\right)^{6} \\
& \cdot \exp \left[-\frac{\varepsilon}{4}\left(\frac{b}{r_{\mathrm{C}}}\right)^{2}\right] .
\end{aligned}
$$

The normalized collision frequency, hence, formally reads

$v_{\mathrm{B}}(B, \varepsilon)=\frac{8}{\ln \Lambda} \frac{(1+2 \varepsilon)^{5 / 2}}{\varepsilon^{3}}\left(\frac{r_{\mathrm{C}}}{b}\right)^{6} \exp \left[-\frac{\varepsilon}{4}\left(\frac{b}{r_{\mathrm{C}}}\right)^{2}\right]$,

valid for $b / r_{\mathrm{C}} \gg 1$. Hence, the exponential decay of $v_{\mathrm{B}}$ is not a signature of the strong interaction approximation [10]. 
Cutting the Coulomb force in different ways, hence, results in different asymptotic formulas for the equipartition frequency. Comparing both expressions (41, 46) for nearly isotropic temperatures $(\varepsilon \approx 1)$, we get

$$
\frac{v_{\mathrm{B}}(B, 1)}{v_{\mathrm{A}}(B, 1)} \approx 8\left(\frac{r_{\mathrm{C}}}{b}\right)^{2} \exp \left[-\frac{1}{4}\left(\frac{b}{r_{\mathrm{C}}}\right)^{2}\right] \text {. }
$$

In view of $b / r_{\mathrm{C}} \gg 1$ this is an extremely small value. Hence, the more stringent isotropic constraint on the Coulomb force restricts the energy exchange to a negligible level.

\section{Comparison with Experiments}

Let us now have a first look on our formal results from a weak interaction Landau theory and on the results obtained in [10] in the ultra strong magnetic field region III, where $r_{\mathrm{C}} / b \ll 1$, being aware that the weak interaction approximation is not justified in this region as discussed in the introduction. The three asymptotic curves are shown in Figure 1.

The solid curve represents (28) of [10], while the dotted line shows the algebraic behavior of the cylindrically cut-off collision frequency (41), and the dashed line is a plot of the spherically cut-off $v,(46)$. All curves are drawn for $\varepsilon \approx 1$. The analytic expressions for the curves are as follows

$$
\begin{aligned}
v & \sim 1.5 \sqrt{6 / \pi} \cdot 0.47 \bar{\varepsilon}^{1 / 5} \exp \left(-2.04 \bar{\varepsilon}^{-2 / 5}\right), \\
v & \sim 3 \sqrt{3} x^{4}, \\
v & \sim 24 \sqrt{3} x^{6} \exp \left(-1 / 4 x^{2}\right),
\end{aligned}
$$

where $x \doteq r_{\mathrm{C}} / b$ and $\bar{\varepsilon} \doteq x /(3 \sqrt{2})$.

Figure 1 very drastically reveals that the weak interaction approximation in this regime does not only yield different results depending on how the Coulomb force is cut off, but also that none of the weak interaction results is able to confirm the experimental results (represented well by the solid line). This is, hence, a cogent evidence of the fact that in the ultra-strong magnetic field region the cumulative small-angle scatterings are less effective.

However, as $r_{\mathrm{c}} / b$ increases and approaches unity, both weak interaction expressions coalesce and at the same time the differences to the experimental results disappear. This can be seen from Fig. 2, which shows the normalized equipartition frequency calculated via (37) and (38) (circles) with $\lambda=10^{7.5}$, together with four experimental data taken from [8] (crosses $\oplus$ ). Experi-

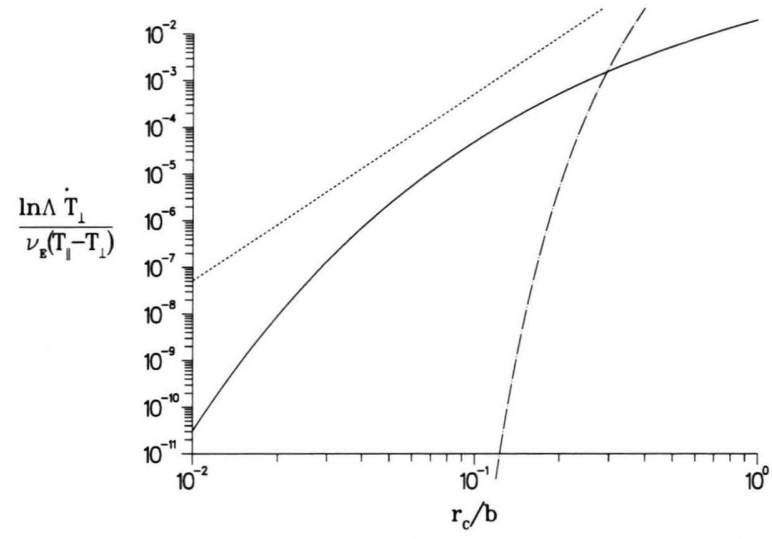

Fig. 1. Asymptotic behavior of the collision frequency for the ultra strong magnetic field region. Solid line: O'Neil et al., dotted line: formal Landau theory with cylindrical cut-off, dashed line: Landau theory with spherical cut-off.

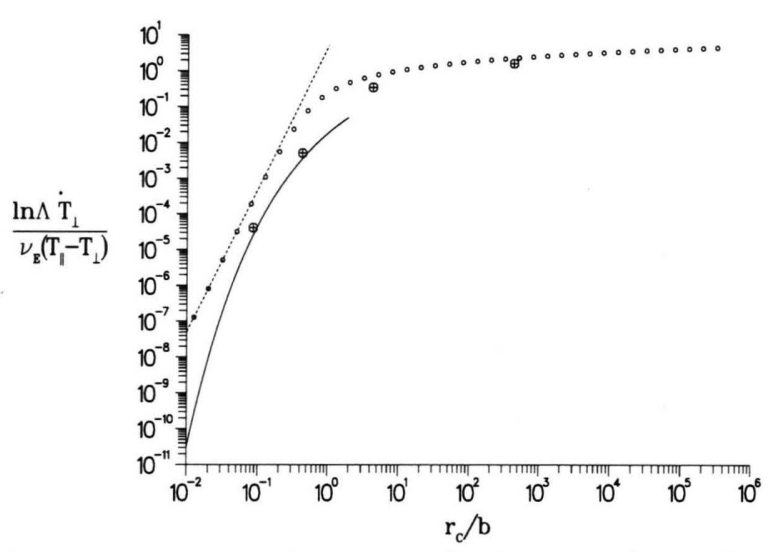

Fig. 2. Isotropisation frequency $v$ for the strong interaction model (solid line) and the weak interaction Landau model (circles o) together with some experimental data (for dotted line, see Fig. 1).

mentally, the temperature range was given by $28 \mathrm{~K}<T<1.1 \cdot 10^{4} \mathrm{~K}$ and the magnetic field strength varied within $3 T<B<6 T$, so that the criterion for a classical treatment, mentioned in the introduction, was satisfied. Furthermore, the asymptotic strong interaction result of [10] (solid line) is shown for $r_{\mathrm{C}} / b<1$, the region of its applicability.

We, therefore, conclude that the transition region $r_{\mathrm{C}} \approx b$, is the boundary between the validity of the strong and weak interaction theory, in agreement with experiment [8] and simulation [13]. 
Table 1. The first few coefficients $a_{k, n}$.

\begin{tabular}{lllllllll}
\hline$n \backslash k$ & 0 & 1 & 2 & 3 & 4 & 5 & 6 & 7 \\
\hline 0 & 1 &. & $\cdot$ &. &. &. & $\cdot$ &. \\
1 & $\cdot$ & 0.25 & -1 & $0 . \overline{3}$ &. & $\cdot$ & \\
2 & $\cdot$ & $\cdot$ & 0.28125 & $-2.41 \overline{6}$ & $2.58 \overline{3}$ & $-0.7 \overline{3}$ & $0.0 \overline{5}$ &. \\
\hline
\end{tabular}

\section{Appendix}

We want to calculate a new form of the asymptotic expansion for the function $e^{-x} I_{l}(x)$ valid for $x \gg l^{2}$ ( $I_{l}$ is the modified Bessel function). Our starting point is the well known asymptotic expansion for fixed $l$, and large $x$ [19].

$$
\begin{aligned}
\sqrt{2 \pi x} & e^{-x} I_{l}(x) \\
& \stackrel{x \rightarrow \infty}{\sim} \sum_{n=0}^{\infty} \frac{(-1)^{n}}{n !(8 x)^{n}} \prod_{k=1}^{n}\left[4 l^{2}-(2 k-1)^{2}\right] \doteq u(l, x) .
\end{aligned}
$$

The series for $u(l, x)$ may now be put into the form

$$
u(l, x)=\sum_{n=0}^{\infty} \frac{\left(\frac{1}{2}+l\right)_{n}\left(\frac{1}{2}-l\right)_{n}}{n !(2 x)^{n}}
$$

where $(z)_{n} \doteq z(z+1) \ldots(z+n-1)$ and $(z)_{0} \doteq 1$.

Defining now the new independent variable $z \doteq$ $1 /(2 x)$, one recognizes that $u(l, z)$ satisfies the differen-

[1] V. P. Silin, Sov. Phys. JETP 14, 617 (1962).

[2] A. Salat and G. Joyce, J. Plasma Phys. 12, 341 (1970).

[3] D. Montgomery, L. Turner, and G. Joyce, Phys. Fluids 17, 954 (1974).

[4] G. Hübner and H. Schamel, Z. Naturforsch. 45a, 1 (1990).

[5] E. Marsch and S. Livi, Ann. Geophysicae 3, 545 (1985).

[6] Ya. L. Al'pert, Space Plasma Vol. 1,2, Cambridge University Press (1990).

[7] B. Beck, Bull. Amer. Phys. Soc. 33, 2004 (1988).

[8] B. Beck, J. Fajans, and J. H. Malmberg, Bull. Amer. Phys. Soc. 35, 2134 (1990).

[9] Wesson, J., Tokamaks, Oxford: Clarendon Press, 1987.

[10] T. M. O'Neil and P. G. Hjorth, Phys. Fluids 28, 3241 (1985).

[11] T. M. O’Neil, Phys. Fluids 26, 2128 (1983).

[12] T. M. O'Neil, P. G. Hjorth, B. Beck, J. Fajans, and J. H. Malmberg, to be published. tial equation

$$
z^{2} u^{\prime \prime}+(2 z-1) \mathrm{u}^{\prime}+\left(\frac{1}{4}-l^{2}\right) u=0 .
$$

Note, that (A-3) can be transformed back into Bessel's equation [20].

We are making now the following (asymptotic) Ansatz for $u$ :

$$
u(l, z)=e^{-l^{2} z} \sum_{n=0}^{\infty} \frac{1}{l^{2 n}} \sum_{k=n}^{3 n} a_{k, n}\left(l^{2} z\right)^{k},
$$

which shall be useful for $l^{2} z \leqq 1$, i.e., $x \geqq l^{2} / 2$.

Substituting (A-4) into (A-3) one gets the recurrence relation for the $a_{k, n}$

$a_{k+1, n+1}=\frac{1}{k+1}\left[a_{k-2, n}-2 k a_{k-1, n}+\left(k+\frac{1}{2}\right)^{2} a_{k, n}\right]$,

$a_{0,0}=1, n=0,1,2, \ldots, k=0, \ldots, 3 n+2, a_{k, n} \equiv 0$ for $k<n$.

The first few values for the coefficients $a_{k, n}$ are given in Table 1.

Resubstituting $x=1 /(2 z)$, we arrive at the final result

$\sqrt{2 \pi x} e^{-x} I_{l}(x) \stackrel{x \rightarrow \infty}{\sim} e^{-l^{2} /(2 x)} \sum_{n=0}^{\infty} \frac{1}{l^{2 n}} \sum_{k=n}^{3 n} a_{k, n}\left(\frac{l^{2}}{2 x}\right)^{k}$.

Retaining only the $n=0$ term, one gets the expression used for deriving (39).

[13] M. E. Glinsky, T. M. O’Neil, M. N. Rosenbluth, K. Tsuruta, and S. Ichimaru, Bull. Amer. Phys. Soc. 35, 2134 (1990).

[14] N. Rostoker, Phys. Fluids 3, 922 (1960).

[15] F. L. Hinton, in: Handbook of Plasma Physics, Volume 1. North Holland: Amsterdam 1983.

[16] H. Schamel, H. Hamnén, D. F. Düchs, T. E. Stringer, and M. R. O'Brien, Phys. Fluids B 1, 76 (1989).

[17] M. H. A. Hassan, Plasma Phys. 19, 1043 (1977).

[18] G. Hübner, Diplomarbeit at University of Bayreuth (1989).

[19] M. Abramowitz and I. A. Stegun, Handbook of Mathematical Functions, Dover Publications, 1970.

[20] E. Kamke, Differentialgleichungen, Akademische Verlagsgesellschaft, Leipzig 1967. 